\title{
Towards a task-driven framework for multimodal fatigue analysis during physical and cognitive tasks
}

\author{
Konstantinos Tsiakas \\ Department of Psychiatry \\ Yale University \\ konstantinos.tsiakas@yale.edu \\ James C. Ford \\ Department of Psychiatry \\ Geisel School of Medicine, Dartmouth \\ james.c.ford@dartmouth.edu
}

\author{
Michalis Papakostas \\ Computer Science and Engineering Department \\ University of Texas at Arlington \\ michalis.papakostas@mavs.uta.edu \\ Fillia Makedon \\ Computer Science and Engineering Department \\ University of Texas at Arlington \\ makedon@uta.edu
}

\begin{abstract}
This paper outlines the development of a task-driven framework for multimodal fatigue analysis during physical and cognitive tasks. While fatigue is a common symptom across several neurological chronic diseases, such as multiple sclerosis (MS), traumatic brain injury (TBI), cerebral palsy (CP) and others, it remains poorly understood, due to various reasons, including subjectivity and variability amongst individuals. Towards this end, we propose a task-driven data collection framework for multimodal fatigue analysis, in the domain of MS, combining behavioral, sensory and subjective measures, while users perform a set of both physical and cognitive tasks, including assessment tests and Activities of Daily Living (ADLs).
\end{abstract}

\section{CCS CONCEPTS}

- Human-centered computing; • Computing methodologies;

\section{KEYWORDS}

Human monitoring, Computer Vision, Human-Computer Interaction, Fatigue Estimation, Cognitive and Physical Training

\section{ACM Reference Format:}

Konstantinos Tsiakas, Michalis Papakostas, James C. Ford, and Fillia Makedon. 2018. Towards a task-driven framework for multimodal fatigue analysis during physical and cognitive tasks. In 5th international Workshop on Sensor-based Activity Recognition and Interaction (iWOAR '18), September 20-21, 2018, Berlin, Germany. ACM, New York, NY, USA, 3 pages. https: //doi.org/10.1145/3266157.3266222

\section{INTRODUCTION}

Multiple sclerosis (MS) is one of the most common neurological chronic diseases affecting primarily young adults between the ages of 20 to 40 years old [1]. The disease comes with a wide range of potential symptoms, which usually significantly affects vision, arm or leg movement, sensation and balance. Patients suffering from MS usually face significant difficulties in walking, numbness or tingling

Permission to make digital or hard copies of part or all of this work for personal or classroom use is granted without fee provided that copies are not made or distributed for profit or commercial advantage and that copies bear this notice and the full citation on the first page. Copyrights for third-party components of this work must be honored.

For all other uses, contact the owner/author(s).

iWOAR '18, September 20-21, 2018, Berlin, Germany

(c) 2018 Copyright held by the owner/author(s).

ACM ISBN 978-1-4503-6487-4/18/09.

https://doi.org/10.1145/3266157.3266222 in different parts of the body, muscle stiffness or spasms and problems with balance and co-ordination, as well as thinking, learning, decision making and planning. In addition, fatigue is one of the most disabling features, since patients need to put significantly more intense efforts towards achieving simple every-day tasks [3]. However, it remains poorly understood due to subjectivity and variability across individuals, as well as different cultural and educational background amongst clinicians and caregivers [6]. Based on the literature, there are three different types of fatigue: physical, emotional and cognitive/mental [12], each one with different effects on patient's quality of life.

As a result, there is a great amount of research focusing on diagnosing such symptoms in their earliest possible stage and tracking their progress over time, during activities of daily living (ADL) and specifically designed assessment tasks [13, 14]. Emerging sensor, wearable and other technologies have opened new opportunities towards making such approaches feasible, by providing both quantitative and qualitative performance metrics that can assist both caregivers and patients towards personalized treatment and rehabilitation. In addition, since fatigue is a common symptom across various mental diseases, these kind of sensor-based approaches have shown great robustness towards modeling behaviors related to different types of neurological disabilities [15, 21].

In this work, we propose a multimodal framework for data collection towards assessing and analyzing fatigue combining both objective and subjective reporting mechanisms, emphasizing on implicit self-reporting mechanisms through the use of sensors. In particular, we propose a task-driven approach which aims to extract both cognitive and physical behavioral patterns which may signal physical and/or mental fatigue, while the user is involved in a set of different tasks. The proposed framework combines different measures extracted through non-invasive sensors, focusing on associating these data with self-reporting mechanisms.

The final outcome of the proposed framework will be a fully annotated multimodal dataset of behavioral and physiological data during traditional cognitive and physical tasks that are already used by domain experts in assessing most of the aforementioned disabilities [4, 22]. Such dataset can provide important insights towards mental and physical fatigue analysis, as well as their impact on user's performance during assessment and rehabilitation tasks, as well as ADLs. 


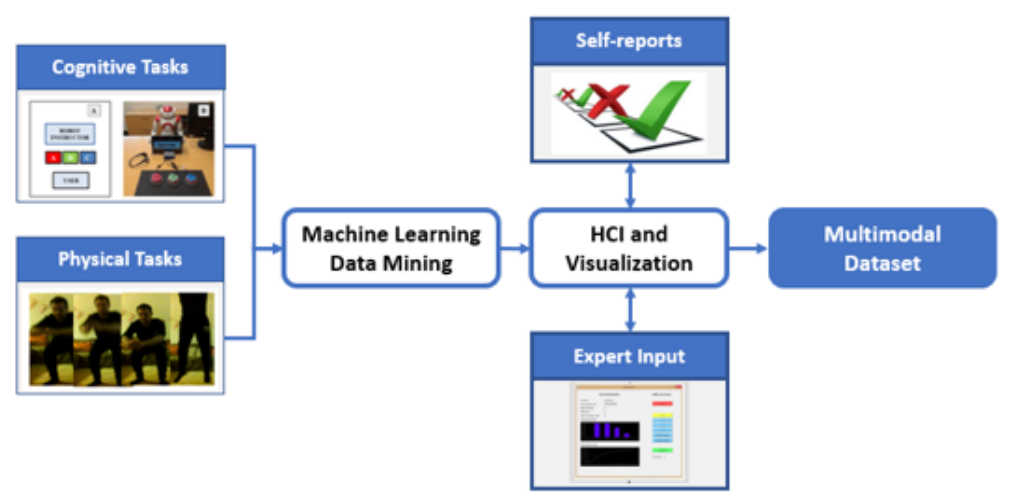

\begin{tabular}{c|c|c|c}
\hline Task Parameters & User Behavior & Physiological Measures & Subjective Measures \\
\hline Task Name & Task Performance & EEG & Self-Report (Difficulty) \\
Task Type & Reaction Time & EMG & Self-Report (Fatigue) \\
Task Difficulty & Completion Time & Heart Rate & Self-Report (Performance) \\
Task Duration & Errors & $E C G$ & Expert Recommendations
\end{tabular}

Figure 1: The proposed framework for multimodal data collection during cognitive and physical tasks for fatigue analysis. Below, an example of possible data captured in the context of our proposed framework. We aim to combine data related to (a) Task parameters, (b) User behavior, (c) Physiological measures, and (d) Subjective measures, for multimodal fatigue analysis.

\section{RELATED WORK AND MOTIVATION}

Physical activity monitoring systems have been proposed towards assessing physical fatigue, either based on task-based physical performance using motion and physiological sensors [10] or by evaluating user performance in ADLs using both objective and self-reporting methods [19]. There has been an increasing use of multiple wearable devices for analyzing and quantifying physical performance and fatigue, associated with specific chronic diseases and their expected behavioral patterns $[2,8,11,17]$.

Towards this end, different sensing modalities have been explored using various types of sensors such as camera-based approaches, electromyography (EMG), heart rate and galvanic skin response (GSR). On the other hand, electroencephalography (EEG) analysis has been the major instrument of assessing cognitive fatigue and related research has shown that it is highly correlated with various neurological impairments like MS $[5,9,16]$. Such approaches have been also exploited on understanding learning abilities and cognitive workload patterns, towards designing adaptive and user-centric assessment tools [7, 18].

To our knowledge, associating subjective and objective measures to analyze both physical and cognitive fatigue to develop reliable fatigue measurement tools has not been properly explored [20]. Our work is motivated by the current need of understanding the extend to which mental and physical fatigue is perceived by different individuals, including both patients and caregivers, as well as the amount to which it affects user behavior and performance. In the following sections we present our proposed framework, which includes different types of multimodal data, in the context of different task-based evaluations.

This material is based upon work supported by the National Science Foundation under Grant No. 1719031 and 1565328 .

\section{PROPOSED FRAMEWORK}

In this section, we present our proposed framework towards developing a multi-modal dataset for mental and physical fatigue analysis. The architecture of the proposed framework is shown in Figure 1. The main goal of the proposed framework is to develop methods and models for multimodal fatigue analysis and detection during physical and cognitive tasks.

For the development of the framework, we follow a task-driven approach; data will be collected during both physical and cognitive tasks, specifically designed to extract behavioral patterns related to fatigue, as well as its effects on human performance. Task-related metrics (e.g., difficulty level) will be combined with behavioral and physiological data. This integration will lead to a multimodal dataset which can be used to analyze user behavior (task performance, reaction time, etc.) under different types of tasks (cognitive vs. physical), different task parameters (task difficulty, task duration), including self-reports from both patients and caregivers/experts.

Since fatigue, as well as its impact to user behavior and performance, is highly subjective and varies across users, we propose to investigate and apply Interactive Machine Learning approaches in order to integrate subjective measures to the development of the proposed framework and dataset. Such methods will be used to enable primary (patients) and secondary (caregivers) users to provide self-reports and expert input which can be used to annotate chunks of the collected dataset. Such a task-driven multimodal dataset may include: (a) task parameters (e.g., task difficulty, task duration) (b) human behavior (e.g., task performance, completion time, errors), (c) physiological data (EEG, EMG, heart rate, etc.) and (d) self-reports and expert input, including subjective measures on task difficulty, fatigue, and performance, as well as expert recommendations, considering appropriate task parameters and targeted interventions for each specific individual. 


\section{REFERENCES}

[1] 2018. Multiple Sclerosis Information Page - NIH National Institute of Neurological Disorders and Stroke - Online. https: //www.ninds.nih.gov/disorders/all-disorders/multiple-sclerosis-information-page

[2] Ashrant Aryal, Ali Ghahramani, and Burcin Becerik-Gerber. 2017. Monitoring fatigue in construction workers using physiological measurements. Automation in Construction 82 (2017), 154-165.

[3] Rohit Bakshi. 2003. Fatigue associated with multiple sclerosis: diagnosis, impact and management. Multiple Sclerosis fournal 9, 3 (2003), 219-227.

[4] Fiona Barwick, Peter Arnett, and Semyon Slobounov. 2012. EEG correlates of fatigue during administration of a neuropsychological test battery. Clinical Neurophysiology 123, 2 (2012), 278-284.

[5] Jason A Berard, Andra M Smith, and Lisa AS Walker. 2018. Predictive Models of Cognitive Fatigue in Multiple Sclerosis. Archives of Clinical Neuropsychology (2018).

[6] Tiffany J Braley and Ronald D Chervin. 2010. Fatigue in multiple sclerosis: mechanisms, evaluation, and treatment. Sleep 33, 8 (2010), 1061-1067.

[7] Chin-Ling Chen, Chong-Yan Liao, Rung-Ching Chen, Yung-Wen Tang, and Tzay Farn Shih. 2017. Bus Drivers Fatigue Measurement Based on Monopolar EEG. In Asian Conference on Intelligent Information and Database Systems. Springer, 308-317.

[8] Emil Chiauzzi, Carlos Rodarte, and Pronabesh DasMahapatra. 2015. Patientcentered activity monitoring in the self-management of chronic health conditions. BMC medicine 13, 1 (2015), 77.

[9] Marina Fiene, Katharina S Rufener, Maria Kuehne, Mike Matzke, Hans-Jochen Heinze, and Tino Zaehle. 2018. Electrophysiological and behavioral effects of frontal transcranial direct current stimulation on cognitive fatigue in multiple sclerosis. Fournal of Neurology (2018), 1-11.

[10] Patty S Freedson and Kelly Miller. 2000. Objective monitoring of physical activity using motion sensors and heart rate. Research quarterly for exercise and sport 71 sup2 (2000), 21-29.

[11] Barry French. 2015. Wearable sensor system with gesture recognition for measuring physical performance. US Patent 9,008,973.

[12] Karla Gonzalez, Farzan Sasangohar, Ranjana K Mehta, Mark Lawley, and Madhav Erraguntla. 2017. Measuring Fatigue through Heart Rate Variability and Activity Recognition: A Scoping Literature Review of Machine Learning Techniques. In Proceedings of the Human Factors and Ergonomics Society Annual Meeting, Vol. 61 SAGE Publications Sage CA: Los Angeles, CA, 1748-1752.
[13] Ludwig Kappos, Mark S Freedman, Chris H Polman, Gilles Edan, Hans-Peter Hartung, David H Miller, Xavier Montalbán, Frederik Barkhof, Ernst-Wilhelm Radü, Lars Bauer, et al. 2007. Effect of early versus delayed interferon beta-1b treatment on disability after a first clinical event suggestive of multiple sclerosis: a 3-year follow-up analysis of the BENEFIT study. The Lancet 370, 9585 (2007), 389-397.

[14] Lauren B Krupp, Nicholas G LaRocca, Joanne Muir-Nash, and Alfred D Steinberg. 1989. The fatigue severity scale: application to patients with multiple sclerosis and systemic lupus erythematosus. Archives of neurology 46, 10 (1989), 1121-1123.

[15] Rajesh Kumar, Parveen Kalra, and AK Lall. 2018. Mental Fatigue Quantification by Physiological and Neurophysiological Techniques: An Overview. In Ergonomics in Caring for People. Springer, 327-336.

[16] Monicque M Lorist, Eniko Bezdan, Michael ten Caat, Mark M Span, Jos BTM Roerdink, and Natasha M Maurits. 2009. The influence of mental fatigue and motivation on neural network dynamics; an EEG coherence study. Brain Research 1270 (2009), 95-106.

[17] Zahra Sedighi Maman, Mohammad Ali Alamdar Yazdi, Lora A Cavuoto, and Fadel M Megahed. 2017. A data-driven approach to modeling physical fatigue in the workplace using wearable sensors. Applied ergonomics 65 (2017), 515-529.

[18] Pasquale Marsella, Alessandro Scorpecci, Giulia Cartocci, Sara Giannantonio, Anton Giulio Maglione, Isotta Venuti, Ambra Brizi, and Fabio Babiloni. 2017. EEG activity as an objective measure of cognitive load during effortful listening: A study on pediatric subjects with bilateral, asymmetric sensorineural hearing loss. International journal of pediatric otorhinolaryngology 99 (2017), 1-7.

[19] F Pitta, Thierry Troosters, VS Probst, MA Spruit, Marc Decramer, and Rik Gosselink. 2006. Quantifying physical activity in daily life with questionnaires and motion sensors in COPD. European respiratory journal 27, 5 (2006), 1040-1055.

[20] Dimitri van der Linden. 2011. The urge to stop: The cognitive and biological nature of acute mental fatigue. Cognitive fatigue: Multidisciplinary perspectives on current research and future applications (2011), 149-64.

[21] Arth Vyas, Arun Gopinathan, Manasa Nimmagadda, Papakostas Michalis, and Fillia Makedon. 2016. CPLAY2: An HCI Game System for the Assessment and Intervention of Children with Cerebral Palsy. In Proceedings of the 9th ACM International Conference on PErvasive Technologies Related to Assistive Environments. ACM, 91.

[22] Philip David Zelazo, Jacob E Anderson, Jennifer Richler, Kathleen Wallner-Allen, Jennifer L Beaumont, and Sandra Weintraub. 2013. II. NIH Toolbox Cognition Battery (CB): Measuring executive function and attention. Monographs of the Society for Research in Child Development 78, 4 (2013), 16-33. 